\title{
Restauración ecológica de bosques tropicales en Costa Rica: efecto de varios modelos en la producción, acumulación y descomposición de hojarasca
}

\author{
Danielle Celentano $^{1 *}$, Rakan A. Zahawi ${ }^{2}$, Bryan Finegan ${ }^{1}$, Fernando Casanoves ${ }^{1}$, Rebecca \\ Ostertag $^{3}$, Rebecca J. Cole ${ }^{4} \&$ Karen D. Holl ${ }^{5}$ \\ 1. Centro Agronómico Tropical de Investigación y Enseñanza (CATIE). Apartado Postal 7170, Turrialba, Cartago, Costa \\ Rica; danielle@catie.ac.cr, bfinegan@catie.ac.cr, casanoves@catie.ac.cr \\ 2. Organización para Estudios Tropicales (OET). Estación Biológica Las Cruces. Apartado Postal 73-8257, San Vito de \\ Coto Brus, Costa Rica; zak.zahawi@ots.ac.cr \\ 3. Universidad de Hawai en Hilo. HI 96720, Hawai, EEUU; ostertag@hawaii.edu \\ 4. Universidad de Hawai en Manoa, Honolulu, HI 96822, EEUU; cole.rebeccaj@gmail.com \\ 5. Universidad de California en Santa Cruz. CA 95064, California, EEUU; kholl@ucsc.edu
}

Recibido 12-X-2010. C Corregido 03-II-2011. Aceptado 01-III-2011.

\begin{abstract}
Tropical forest restoration in Costa Rica: the effect of several strategies on litter production, accumulation and decomposition. Tropical forest restoration strategies have the potential to accelerate the recovery of the nutrient cycles in degraded lands. Litter production and its decomposition represent the main transfer of organic material and nutrients into the soil substrate. We evaluated litter production, accumulation on the forest floor, and its decomposition under three restoration strategies: plantation (entire area planted with trees), island (trees planted in patches of three different sizes) and control (natural regeneration) plots. We also compared restoration strategies to young secondary forest (7-9yr). Restoration treatments were established in 50x50m plots in June 2004 at six sites in Southern Costa Rica. Planted tree species included two native timber species (Terminalia amazonia and Vochysia guatemalensis) interplanted with two N fixers (Erythrina poeppigiana and Inga edulis). Litter was collected every 15 days between September 2008 and August 2009 in 12 $0.25 \mathrm{~m}^{2}$ litter traps distributed within each plot; litter that accumulated on the soil surface was collected at four locations $\left(0.25 \mathrm{~m}^{2}\right.$ quadrats $)$ within each plot in February and May 2009. Total litter production in plantation $(6.3 \mathrm{Mg} / \mathrm{ha})$ and secondary forest $(7.3 \mathrm{Mg} / \mathrm{ha})$ did not differ, but were greater than in islands $(3.5 \mathrm{Mg} / \mathrm{ha})$ and control $(1.4 \mathrm{Mg} / \mathrm{ha})$. Plantation had greatest accumulation of litter on the soil surface $(10.6 \mathrm{Mg} / \mathrm{ha})$ as compared to the other treatments $(\mathrm{SF}=7.2 ; \mathrm{I}=6.7 ; \mathrm{C}=4.9)$. Secondary forest was the only treatment with a greater annual production of litter than litter accumulation on the soil surface. Carbon storage in litter was similar between plantation and secondary forest, and significantly greater than the other treatments. No differences were found for carbon concentration and storage in the soil among treatments. There was also high variability in the production and accumulation of litter and carbon among sites. Active restoration treatments accelerated the production of litter and carbon storage in comparison to areas under natural recovery. However, the nutrient cycle has not necessarily been restored under these conditions, as high litter accumulation on the soil surface indicates a low decomposition rate, which slows nutrient return to the soil. Rev. Biol. Trop. 59 (3): 1323-1336. Epub 2011 September 01.
\end{abstract}

Key words: tropical forest, restoration, plantation, natural succession, litterfall, carbon, decomposition.

Los bosques tropicales protegen la mayor diversidad biológica del planeta y brindan servicios ecosistémicos vitales. No obstante, la mayor parte de esos bosques están sostenidos por suelos de moderada a baja fertilidad
(Vitousek \& Sanford 1986). Bajo esas condiciones, la biomasa sostiene una importante proporción de los nutrientes que están potencialmente disponibles para la biota, y las plantas han desarrollado mecanismos altamente 
adaptados para la adquisición y la retención de los nutrientes (Walker \& Reddell 2007). Además de alterar la estructura del ecosistema y arrasar con el capital natural, la deforestación altera los ciclos de nutrientes y aumenta la fuga de nutrientes del ecosistema (Walker \& Reddell 2007) con altos costos ambientales y sociales (MEA 2005). No obstante, la deforestación sigue siendo intensa en la mayoría de los países tropicales, especialmente en América Latina, donde los bosques son talados y rápidamente convertidos para la agricultura (FAO 2006). Así, las estrategias de restauración en los trópicos son indispensables considerando la gran escala de degradación (Lamb et al. 2005) y la necesidad de mantener procesos ecológicos vitales.

Restauración ecológica se define como el proceso intencional de ayudar al restablecimiento de un ecosistema que ha sido degradado, dañado o destruido (SERI 2004). Este proceso incluye el secuestro de carbono de la atmósfera, la recuperación de hábitat para la biodiversidad y la provisión de bienes y servicios ecosistémicos (Chazdon 2008). Además de restablecer la integridad ecológica, la restauración representa una manera de mejorar el bienestar humano en paisajes degradados a través de la recuperación de la productividad de la tierra y del capital natural (ITTO \& IUCN 2005, Mansourian et al. 2005, Aronson et al. 2007). Los principios de la restauración ecológica de ecosistemas terrestres son los mismos que los principios de la sucesión ecológica (Bradshaw 1987). La meta final del proceso no es recuperar integralmente el estado anterior al disturbio, pero si garantizar que las funciones y los procesos ecológicos sean similares al ecosistema original a través del tiempo (SERI 2004). Según Lamb \& Gilmour (2003), en el futuro tal vez sea apropiado buscar la restauración de vegetación natural resiliente y que presente rasgos funcionales para el restablecimiento de algunos servicios ecosistémicos deseados. Diferentes métodos han sido propuestos para acelerar o facilitar la restauración en los trópicos desde el punto de vista ecológico (Lamb \& Gilmour 2003, Lamb et al. 2005). La selección del método más apropiado depende del estado inicial de la degradación, los resultados deseados y del presupuesto disponible (Chazdon 2008); además del entorno socio-cultural y el marco político.

Desde el punto de vista ecológico, el método más sencillo de restauración es eliminar la fuente de perturbación y permitir al ecosistema recuperarse naturalmente (conocido también como restauración pasiva). Ese método es el más indicado cuando los recursos financieros son escasos y/o el estado de degradación no es excesivo. En este caso, es necesario proteger la zona de cualquier tipo de perturbación externa (incendios, ganado, etc.), de manera que los procesos de regeneración ocurran naturalmente (Lamb \& Gilmour 2003). Prach et al. (2007) sostienen que la sucesión natural es el método que resulta en una mayor diversidad estructural y funcional del ecosistema. No obstante, el abandono no siempre dará lugar al desarrollo sucesional deseado (Schrautzer et al. 2007). Muchos factores bióticos y abióticos afectan el restablecimiento del bosque en un área alterada. La ausencia de dispersores de semillas, la falta de nutrientes en los suelos y la competencia de plántulas con gramíneas agresivas son considerados obstáculos importantes (Uhl et al. 1988, Nepstad et al. 1996, Holl 2002). Manipulaciones del ambiente físico y de la biota pueden reducir el tiempo necesario para la sucesión (Prach et al. 2007).

La plantación de árboles en alta densidad es el método más común de restauración activa. Las especies plantadas pueden establecerse rápidamente, suprimir las gramíneas y crear mejores condiciones para el establecimiento de una comunidad más diversa (Reay \& Norton 1999). Sin embargo, plantaciones densas presentan altos costos iniciales y mucha inversión en cuanto a mantenimiento (Lamb \& Gilmour 2003). Considerando que la mayoría de las iniciativas de restauración tienen presupuesto restringido (Holl \& Howarth 2000), técnicas menos costosas pueden ser más atractivas para los agricultores, como por ejemplo la siembra de "islas de árboles", las cuales tienen como objetivo crear una complejidad estructural que 
imita el proceso de regeneración natural conocido como nucleación, donde parches de vegetación sucesional crean microhábitat favorables para el establecimiento de especies tardías (Yarranton \& Morison 1974). Ese método requiere menor cantidad de árboles por hectárea y es más económico que las plantaciones tradicionales. Además de la restauración de hábitat para la biodiversidad, los métodos activos tienen el potencial para acelerar el restablecimiento de procesos ecológicos como los ciclos de nutrientes y el secuestro de carbono. Este supuesto puede ser abordado a través del estudio de la dinámica de la hojarasca.

La producción de hojarasca y su descomposición son procesos fundamentales en el ciclo de nutrientes, ya que representan la principal transferencia de materia orgánica y nutrientes desde la vegetación a la superficie del suelo (Vitousek \& Sanford 1986). La hojarasca es una medida de producción primaria neta (PPN) del ecosistema y está fuertemente correlacionada con el incremento de la biomasa (Clark et al. 2001), la densidad de árboles y la apertura del dosel (Zou et al. 1995, Oelbermann \& Gordon 2000), siendo afectada por variables ambientales como la precipitación, la temperatura, la elevación y la fertilidad de los suelos (Vitousek \& Sanford 1986) y la evapotranspiración potencial (Meentemeyer et al. 1982). La producción y acumulación de hojarasca se incrementa rápidamente en los primeros años de sucesión (Ewel 1976). No obstante, después que el dosel está cerrado, no hay una tendencia clara entre la producción de hojarasca y la edad del bosque (Ewel 1976, Lugo 1992, Zou et al. 1995, Ostertag et al. 2008), así como con la riqueza y la diversidad de especies (Wardle et al. 1997, Scherer-Lorenzen et al. 2007).

La composición de especies determina las características químicas de la hojarasca y afecta el proceso de descomposición (Zou et al. 1995, Xuluc-Tolosa et al. 2003). El proceso de descomposición de la hojarasca implica una serie de interacciones entre la materia orgánica, los micro-organismos del suelo, las comunidades de invertebrados, y las condiciones ambientales. Las comunidades de micro-organismos y fauna en el suelo son influenciadas por la cantidad y la calidad de la hojarasca acumulada sobre el suelo y por su distribución espacial (Schroth 2003). Esas interacciones son muy afectadas en áreas degradadas, donde hay bajo aporte y acumulación de materia orgánica y cambios en las características ambientales. Por lo tanto, se espera que estas interacciones sean restablecidas durante el proceso de restauración, con el incremento en el aporte y acumulación de materia orgánica.

En este estudio se evaluó la hojarasca y el carbono almacenado en el suelo en un proyecto de restauración forestal llevado a cabo en el cantón de Coto Brus, sur de Costa Rica (Holl et al. 2011). El proyecto compara la regeneración natural con dos estrategias de restauración activa (plantación mixta de árboles y plantación de “islas de árboles") que pueden ser un modelo viable para otras regiones tropicales. Ambos sistemas de restauración utilizan dos especies nativas maderables (Terminalia amazonia (J.F. Gmel.) Exell y Vochysia guatemalensis Donn. Sm.), intercaladas con dos especies introducidas fijadoras de nitrógeno (Erythrina poeppigiana (Walp.) O. F. Cook e Inga edulis Mart.) que han demostrado aumentar la disponibilidad de nutrientes en el suelo y mejorar las condiciones para el establecimiento de plántulas (Nichols et al. 2001, Nichols \& Carpenter 2006). El objetivo específico fue evaluar la producción, acumulación en el suelo y descomposición de la hojarasca y el carbono almacenado en la hojarasca y suelo mediante dos métodos de restauración activa y compararlos con áreas en regeneración natural y bosques secundarios jóvenes. Nuestra hipótesis es que después de cinco años: (1) las dos estrategias de restauración activas presentarán más producción y acumulación de hojarasca, mayor contenido de carbono en el suelo y tasas de descomposición más bajas que las áreas en regeneración natural, debido a una mayor densidad de árboles y a una menor apertura del dosel; y (2) las plantaciones y los bosques secundarios jóvenes no presentarán diferencias en la producción, acumulación y descomposición de hojarasca y de carbono en el suelo como consecuencia 
de presentar similar densidad de árboles y apertura del dosel.

\section{MATERIALES Y MÉTODOS}

Área de estudio: El estudio se llevó a cabo en seis sitios ubicados entre la Estación Biológica Las Cruces (847'7" N-82 57'32" W) y la ciudad de Agua Buena ( $8^{\circ} 44^{\prime} 42^{\prime}$ " N-82 56'53" W) en Coto Brus, Pacífico sur de Costa Rica. El área de estudio es un paisaje agrícola fragmentado localizado en el Corredor Biológico Amistosa - un área prioritaria para la conservación en Costa Rica, ya que tiene por objetivo conectar los mayores remanentes de bosques primarios en el sur del país (Céspedes et al. 2008). En los últimos 50 años la mayor parte de la zona de estudio fue deforestada y convertida a uso agrícola (especialmente café). El café fue cambiado a potreros o abandonado después de una importante crisis económica (Rickert 2005). Actualmente menos de $25 \%$ del bosque original está conservado en pequeños fragmentos en esta región (Daily et al. 2001).

Los sitios de estudio fueron abandonados recientemente y habían sido utilizados para la agricultura (pastizales y/o fincas de café) entre 18 y 50 años antes del inicio del experimento. La mayoría fueron quemados una o dos veces después de la conversión. Los sitios eran dominados ( $>80 \%$ de cobertura) por gramíneas
(Pennisetum purpureum Schumach y/o Urochloa brizantha (Hochst. Ex. A. Rich.) R.D. Webster) o una mezcla de forraje, gramíneas, plantas herbáceas y Pteridium arachnoideum (Kaulf.) Maxon. Una descripción detallada de los sitios fue presentada en Cole et al. (2010) y Holl et al. (2011).

Los bosques en la zona se clasifican como bosques muy húmedos premontanos (Holdridge et al. 1975). Los sitios presentan un rango de elevación de 1000 a 1 300m y tienen fuerte pendiente. La temperatura promedio $\left(21^{\circ} \mathrm{C}\right)$ presenta poca variación durante el año y la zona recibe 3 500-4 000mm de precipitación anuales, con una marcada estación lluviosa entre mayo y diciembre (Fink et al. 2009). Los suelos (Inceptisoles) son ácidos ( $\mathrm{pH}$ 5.5, Al Acidez $>0.5 \mathrm{cmol} / \mathrm{kg}$ y la saturación de ácido $>15 \%$ a $5-20 \mathrm{~cm}$ de profundidad), pobres en fósforo $(4.09 \mathrm{mg} / \mathrm{kg}$ a $0-5$ y 1.95 en $5-10 \mathrm{~cm})$, con niveles bajos a moderados de cationes intercambiables (Ca: $9.74 \mathrm{cmol} / \mathrm{kg} \pm 1.54, \mathrm{Mg}$ : $3.41 \pm 0.44, \mathrm{~K}: 0.47 \pm 0.08)$ y concentraciones muy altas de $\mathrm{Fe}(142.15 \mathrm{mg} / \mathrm{kg} \pm 11.84)$. La densidad aparente promedio es de $0.63 \mathrm{~g} / \mathrm{cm}^{3}$ $( \pm 0.03)$ y presenta alto tenor de materia orgánica $(14 \%$ a $0-5 \mathrm{~cm}$ y $11 \%$ a $l o s 5-20 \mathrm{~cm})$.

Diseño experimental y tratamientos: En cada sitio (bloques) se establecieron tres tratamientos en junio de 2004 (Fig. 1). Los tratamientos fueron: Plantación (P: toda la
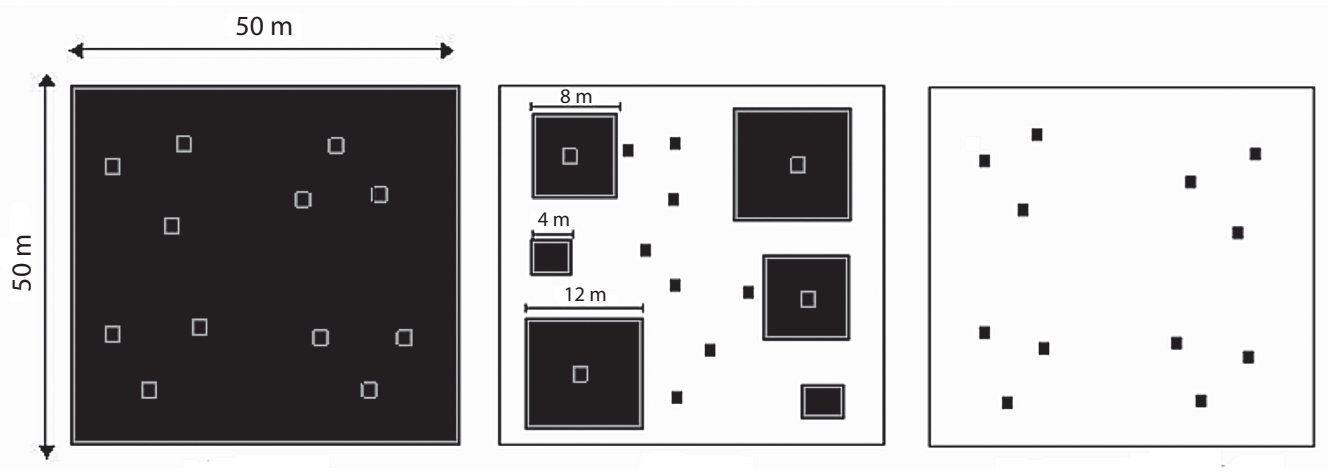

Fig. 1. Diseño de las parcelas: plantaciones (izquierda), islas (centro) y testigo/bosque secundario (derecha). Negro=superficie plantada con árboles. Blanco=zonas no plantadas. Cuadrados pequeños=canastas de recolecta de hojarasca.

Fig. 1. Plot layout: plantation (left), island (middle) and control/secondary forest (right). Black=areas planted with tree seedlings. White=unplanted areas. Small squares=litter traps for litterfall collection. 
superficie plantada con una mezcla de especies arbóreas); Islas (I: árboles sembrados en parches de tres tamaños: $4 x 4 \mathrm{~m}, 8 \mathrm{x} 8 \mathrm{~m}$, y $12 \times 12 \mathrm{~m}$ ); y Testigo (T: sin plantación de árboles/regeneración natural). Las unidades experimentales consistieron en parcelas de 50x50m. Las parcelas fueron plantadas con cuatro especies, siendo dos nativas maderables, Terminalia amazonia (J.F. Gmel.) Exell (Combretaceae), Vochysia guatemalensis Donn. Sm. (Vochysiaceae) y dos especies introducidas de rápido crecimiento y fijadoras de nitrógeno, Erythrina poeppigiana (Walp.) O. F. Cook (Fabaceae), e Inga edulis Mart. (Fabaceae). El desarrollo de los árboles plantados presentó gran variación entre los sitios (Fig. 2, más detalles sobre el desarrollo de los árboles plantados en Holl et al. 2011). En tres de los seis sitios, una parcela adicional de bosque secundario joven (BS: 7-9 años) fue evaluada como un cuarto tratamiento. Los bosques tienen una historia de uso del suelo similar a las demás parcelas y una estructura similar a las plantaciones: (1) altura de los árboles variando entre 5-15m; (2) cobertura del dosel entre 45 a $90 \%$ y (3) número promedio de especies arbóreas por parcela de 7.3 (listado de especies presentado en Celentano et al. 2011). Un total de 21 parcelas fueron utilizadas para el experimento.

\section{Variables}

Producción de hojarasca: En cada una de las 21 parcelas fueron establecidas 12 canastas de recolecta de hojarasca. Las canastas $\left(0.25 \mathrm{~m}^{2}\right.$ de área) fueron puestas aproximadamente a $0.60 \mathrm{~m}$ sobre el nivel del suelo. En el tratamiento de Islas, una canasta fue ubicada dentro de cada isla mediana y grande, dos fueron colocadas dentro de un perímetro de $2 \mathrm{~m}$ de los árboles sembrados, y las restantes fueron ubicadas en áreas sin plantaciones ( $>2 \mathrm{~m}$ de la base de los árboles sembrados; Fig. 1). En los otros tratamientos, grupos de 3 canastas (separadas entre 4-10m) fueron distribuidas en cada uno de los cuatro cuadrantes de la parcelas. La hojarasca fue recogida cada 15 días entre los meses de
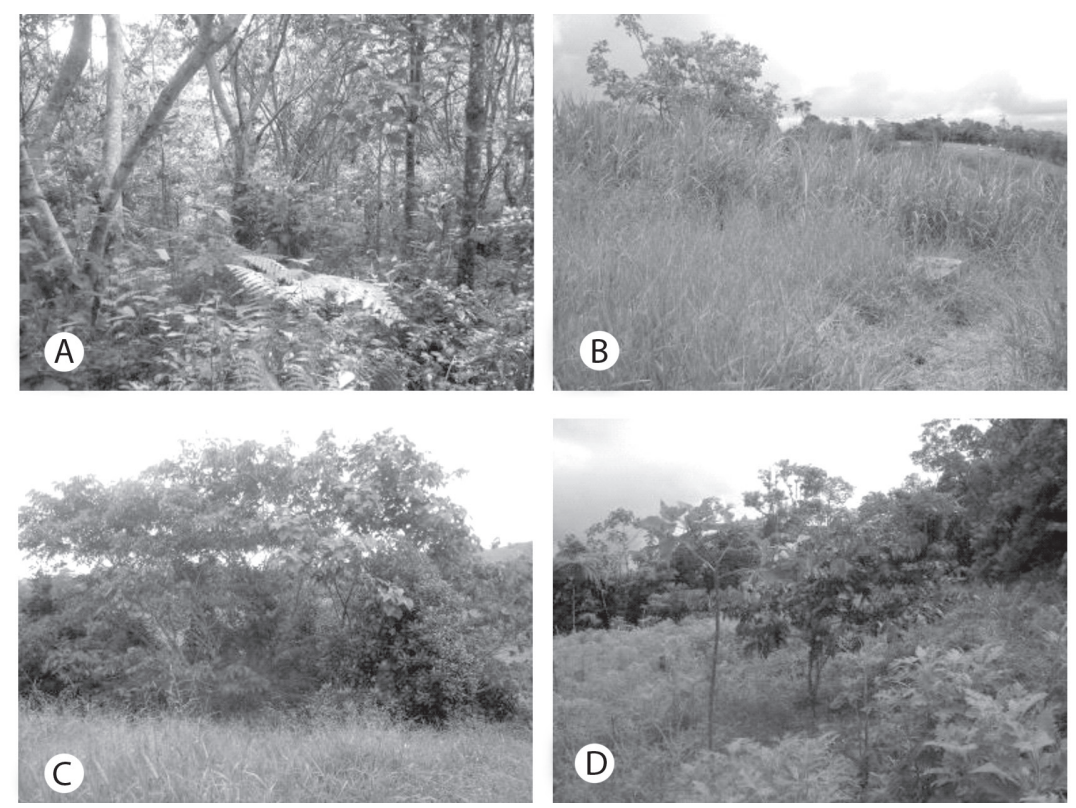

Fig. 2. Fotos de los tratamientos con 4 años de edad (a). Plantación. (b). Testigo. (c). Una Isla Mediana "bien desarrollada". (d). Una isla grande "mal desarrollada".

Fig. 2. Pictures of treatments at 4 years (a). Plantation. (b). Control. (c). A medium "well developed" island. (d). A large "poorly developed" island. 
septiembre 2008 y agosto 2009. Después de secar en un horno a $65^{\circ} \mathrm{C}$ por $24-48 \mathrm{hr}$ (hasta estabilización del peso seco), la masa de hojarasca fue evaluada separadamente en hojas, madera (leños finos de hasta $1 \mathrm{~cm}$ de diámetro), partes reproductivas (flores, frutos y semillas) y misceláneos (material vegetal indeterminado de pequeña dimensión) para cada muestreo. Una vez al mes, se pesaron las hojas clasificándolas por cada una de las cuatro especies sembradas, gramíneas, otras especies dicotiledóneas y material no identificado. Esa identificación no fue llevada a cabo en los Bosques Secundarios (mayores detalles presentados en Celentano et al. 2011).

Hojarasca acumulada sobre el suelo: $\mathrm{La}$ hojarasca almacenada sobre el suelo fue cuantificada en febrero y mayo del 2009 y promediada. Se ubicó un cuadro de PVC de $0.25 \mathrm{~m}^{2}$ en puntos de muestro definidos en forma aleatoria, a razón de ocho en las islas y cuatro en el bosque secundario, la plantación y el testigo). Toda la materia orgánica vegetal acumulada dentro del cuadro fue recogida y el peso seco $\left(65^{\circ} \mathrm{C}\right.$ por 24-48hr hasta estabilización) fue evaluado por separado en: hojas de dicotiledóneas, hojas y tallo de gramíneas, partes reproductivas, madera y otros. Antes de recoger la hojarasca acumulada sobre el suelo, se estimó visualmente su cobertura (\%) y se midió con una regla su espesor $(\mathrm{cm})$.

Carbono de la hojarasca: La concentración de carbono en las hojas fue determinada en el laboratorio de Análisis de Suelos, Tejido Vegetal y Aguas del CATIE (Centro Agronómico Tropical de Investigación y Enseñanza) por el método de combustión en equipo autoanalizador (AOAC 1984) para cuatro períodos de análisis: (1) octubre 2008; (2) muestras compuestas de diciembre, enero y febrero; (3) marzo, abril y mayo; y (4) junio, julio y agosto. La concentración de carbono de los otros componentes de la hojarasca (madera, partes reproductivas y misceláneos) fue estimada como la mitad de la masa seca (Pearson et al. 2005). Con los datos de concentración de carbono y de producción y acumulación, se calcularon el aporte y el almacenamiento de carbono por hectárea en cada tratamiento.

Carbono en el suelo: El carbono del suelo fue muestreado en abril 2009, cuando se recogieron diez muestras al azar en cada parcela a dos profundidades $(0-5$ y $5-20 \mathrm{~cm})$. Las muestras de cada parcela fueron mezcladas, secadas al aire y tamizadas $(2 \mathrm{~mm})$. El análisis químico para determinar la concentración de carbono total se realizó en una única muestra compuesta por parcela siguiendo el protocolo estándar del laboratorio de suelos del CATIE (Díaz \& Hunter 1978). Para determinar el contenido de carbono es necesario conocer la densidad aparente del suelo. Para esto, se recogieron cinco muestras de cada parcela con un diámetro de $4 \times 10 \mathrm{~cm}$ de profundidad. Las muestras de suelo para el cálculo de la densidad aparente se secaron a $105^{\circ} \mathrm{C}$ durante $48 \mathrm{hr}$ antes de su pesaje.

Descomposición: El potencial de descomposición entre los diferentes tratamientos fue evaluado en un experimento preliminar usando el método "standard leaf-litter species" (Upadhyay et al. 1985) que utiliza un material vegetal similar para garantizar una calidad de substrato constante. La descomposición fue estimada usando bolsas de descomposición (litterbags) de $25 \mathrm{~cm}^{2}$ con malla de $2 \mathrm{~mm}$ llenas con $10 \mathrm{~g}$ de hojas secas homogeneizadas de I. edulis, que fue seleccionada por ser la especie que más hojarasca aporta en las parcelas de restauración estudiadas. Las bolsas fueron enterradas superficialmente $(\sim 1-2 \mathrm{~cm}$ de profundidad) en cada parcela (Bocock \& Gilbert 1957). Este estudio fue llevado a cabo en los tres sitios donde hay bosque secundario. El experimento fue instalado en Febrero de 2009 (T0) y la pérdida de peso de las bolsas fue evaluada en cuatro periodos de incubación $(\mathrm{T} 1=\mathrm{T} 0+15$ días, $\mathrm{T} 2=\mathrm{T} 0+30$ días, T3=T0+60 días y T4=T0+135 días). Cuatro bolsas por parcela fueron promediadas para cada período de incubación. En total fueron instaladas 192 bolsas en el campo (12 parcelas $\times 4$ periodos de incubación $\times 4$ repeticiones). 
El experimento se estableció como un diseño de bloques incompletos (el tratamiento de bosque secundario se replicó solamente en tres de los seis sitios) con el sitio como el factor de bloqueo. Las diferencias en la hojarasca (producción total, acumulación y carbono) y el carbono del suelo entre las estrategias de restauración fueron analizadas usando ANOVA en el contexto de los modelos generales y mixtos, con tratamientos como factores fijos y los sitios como factores aleatorios, y las comparaciones entre tratamiento se llevaron a cabo a través del test LSD de Fisher.

El experimento con las bolsas de descomposición se llevó a cabo según un diseño en bloques completos (solo se utilizaron los tres sitios donde existían repeticiones del bosque secundario). Para evaluar la tasa de descomposición, calculamos y graficamos la pérdida de peso de las bolsas de descomposición por tiempo. Después se ajustó la curva de pérdida de peso usando regresiones lineales (Materia seca remanente $=\beta_{0}+\beta_{1}$ Tiempo) y exponenciales (Materia seca remanente $=\beta_{0}$ e $^{\beta 1 \text { Tiempo }}$ y se calculó el coeficiente $k$ de descomposición para cada parcela de acuerdo con el modelo mejor ajustado. Finalmente, se utilizó una ANOVA para hacer la comparación entre los valores del coeficiente $k$ de descomposición entre los tratamientos. Todos los análisis estadísticos y gráficos fueron realizados con InfoStat $/ \mathrm{P}^{\circledR}$ (versión 2009, Di Renzo et al. 2009) y $\mathrm{R}^{\circledR}$ (versión 2.7.2; R Development Core Team 2010).

\section{RESULTADOS}

\section{Producción y acumulación de hojaras-}

ca: La producción anual de hojarasca así como su acumulación sobre el suelo difirió entre los sitios y los tratamientos (Fig. 3). Hubo gran variabilidad entre los sitios en el promedio

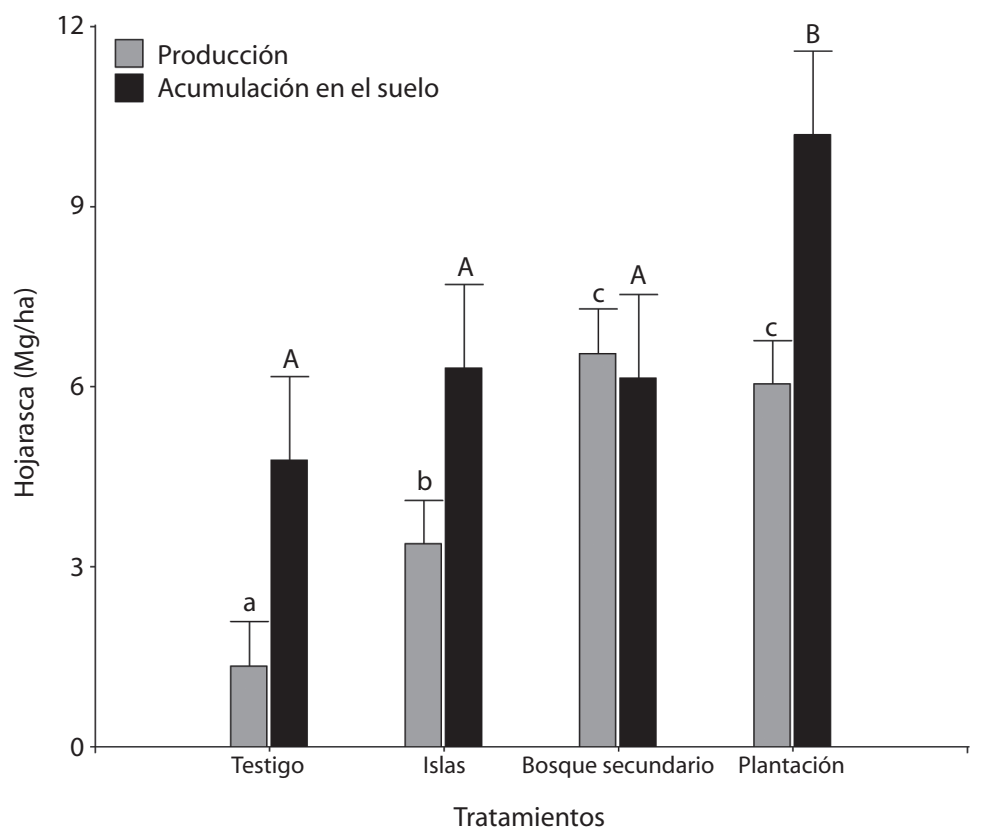

Fig. 3. Hojarasca producida ( $\mathrm{Mg} / \mathrm{ha} / \mathrm{año})$ y acumulada sobre el suelo $(\mathrm{Mg} / \mathrm{ha})$ en los tratamientos de restauración (plantación, islas, testigo y bosque secundario) en Coto Brus, Costa Rica. Letras diferentes indican diferencia estadística $(\mathrm{p}<0.05)$ entre tratamientos de acuerdo con la prueba LSD de Fisher.

Fig. 3. Litterfall production ( $\mathrm{Mg} / \mathrm{ha} / \mathrm{yr})$ and accumulation above the soil $(\mathrm{Mg} / \mathrm{ha})$ in the restoration treatments (plantation, islands, control and secondary forest) in Coto Brus, Costa Rica. Values with the same letter are not significantly different ( $p>0.05$ ) across treatments according to Fisher's LSD. 
de producción (1.1-5.7Mg / ha/año) y acumulación (2.4-10.5Mg/ha) de hojarasca, y dos sitios tuvieron producción $(\mathrm{F}=31.4, \mathrm{p}<0.0001)$ y acumulación $(\mathrm{F}=6.26, \mathrm{p}<0.0001)$ inferior a los demás. El bosque secundario y la plantación presentaron la mayor producción anual de hojarasca, mientras que las islas presentaron valores intermedios y el testigo mostró una producción reducida $(\mathrm{F}=129.35, \mathrm{p}<0.0001)$. A su vez, la hojarasca acumulada en el suelo fue superior en las plantaciones que en los otros tratamientos $(\mathrm{F}=6.51, \mathrm{p}<0.0004)$.

La mayor cobertura de hojarasca en el suelo se presentó en las plantaciones $(97.5 \%)$ y fue estadísticamente superior $(\mathrm{F}=6.34, \mathrm{p}<0.0006)$ a los otros tratamientos $(\mathrm{BS}=83.4 \%, \mathrm{I}=82.8 \%$; $\mathrm{T}=80.6 \%$ ). De igual manera, las plantaciones presentaron una capa de hojarasca $(\mathrm{F}=8.38$, $\mathrm{p}<0.0001)$ con mayor espesor $(3.7 \mathrm{~cm})$, mientras que el bosque secundario $(1.8 \mathrm{~cm})$ y el testigo $(2.1 \mathrm{~cm})$ presentaron capas más delgadas; las islas tuvieron valores intermedios $(2.9 \mathrm{~cm})$. En todos los tratamientos, las hojas fueron el principal componente de la hojarasca producida $(\mathrm{P}=87 \%$ del peso seco, $\mathrm{I}=88 \%$, $\mathrm{T}=89 \%, \mathrm{BS}=77 \%$ ) y de la hojarasca acumulada ( $\mathrm{P}=88 \%, \mathrm{I}=90 \%, \mathrm{~T}=93 \%, \mathrm{BS}=74 \%)$. En los bosques secundarios, la madera representó el $20 \%$ de la biomasa seca de la hojarasca acumulada. La especie que más contribuyó en la producción de hojas en las plantaciones (70\%) e Islas $(47 \%)$ fue I. edulis. Mayores detalles respecto a la producción de hojarasca son presentados en Celentano et al. (2011).

\section{Concentración, aporte y almacenamien-}

to de Carbono: La concentración de carbono en las hojas varió entre tratamientos $(\mathrm{F}=9.83$, $\mathrm{p}=0.0015$ ), siendo el testigo (43\%) quien presentó una concentración de carbono en las hojas estadísticamente inferior a los otros tratamientos $(\mathrm{P}=48 \%, \mathrm{BS}=46 \%, \mathrm{I}=46 \%)$. Como consecuencia de la producción anual de hojarasca, el aporte anual de carbono (Cuadro 1) fue superior en el bosque secundario y en las plantaciones, intermedio en las islas e inferior en el testigo. De igual manera, el carbono almacenado en hojarasca sobre el suelo fue superior en las plantaciones $(4.6 \mathrm{MgC} / \mathrm{ha} / \mathrm{año})$ $\mathrm{y}$ en el bosque secundario (2.9MgC/ha/año). No obstante, el almacenamiento de carbono en hojas fue mayor en la plantación que en todos los otros tratamientos.

\section{CUADRO 1}

Aporte y almacenamiento de carbono en la hojarasca y suelo por hectárea $(\mathrm{Mg} / \mathrm{ha}$, promedio \pm desviación estándar) en los tratamientos de restauración (plantación, islas, testigo y bosque secundario) en Coto Brus, Costa Rica

TABLE 1

Input and storage of carbon from litterfall and soil $(\mathrm{Mg} / \mathrm{ha}$, mean \pm standard deviation) in the restoration treatments (plantation, islands, control and secondary forest) in Coto Brus, Costa Rica

$\begin{array}{lcccccc}\text { Contenido de carbono (Mg C/ ha) } & \text { Testigo } & \text { Islas } & \text { Plantación } & \text { Bosque secundario } & \text { F } & \text { p } \\ \text { Hojarasca producida (año) } & 0.6 \pm 0.4^{\mathrm{a}} & 1.6 \pm 0.4^{\mathrm{a}} & 3.0 \pm 0.4^{\mathrm{b}} & 3.3 \pm 0.6^{\mathrm{b}} & 11.27 & 0.0008 \\ \text { Hojas } & 0.5 \pm 0.4^{\mathrm{a}} & 1.4 \pm 0.4^{\mathrm{b}} & 2.6 \pm 0.4^{\mathrm{c}} & 2.5 \pm 0.5^{\mathrm{bc}} & 11.62 & 0.0007 \\ \text { Otros componentes } & 0.1 \pm 0.1^{\mathrm{a}} & 0.2 \pm 0.1^{\mathrm{ab}} & 0.5 \pm 0.1^{\mathrm{b}} & 0.9 \pm 0.1^{\mathrm{c}} & 8.39 & 0.0028 \\ \text { Hojarasca acumulada } & 1.9 \pm 0.6^{\mathrm{a}} & 2.5 \pm 0.6^{\mathrm{a}} & 4.6 \pm 0.6^{\mathrm{b}} & 2.9 \pm 0.8^{\mathrm{ab}} & 6.29 & 0.0083 \\ \text { Hojas } & 1.8 \pm 0.6^{\mathrm{a}} & 2.2 \pm 0.6^{\mathrm{a}} & 3.9 \pm 0.6^{\mathrm{b}} & 2.1 \pm 0.8^{\mathrm{a}} & 5.09 & 0.0168 \\ \text { Otros componentes } & 0.2 \pm 0.1^{\mathrm{a}} & 0.3 \pm 0.1^{\mathrm{ab}} & 0.6 \pm 0.1^{\mathrm{bc}} & 0.9 \pm 0.2^{\mathrm{c}} & 7.23 & 0.0050 \\ \text { Suelo total (0-20cm) } & 80.6 \pm 8.2 & 75.8 \pm 8.2 & 75.1 \pm 8.2 & 91.9 \pm 9.5 & - & 0.1855 \\ \text { Suelo (0-5cm) } & 24.4 \pm 2.7 & 23.5 \pm 2.7 & 22.9 \pm 2.7 & 27.9 \pm 3.0 & - & 0.1743 \\ \text { Suelo (5-20cm) } & 56.2 \pm 5.7 & 52.4 \pm 5.7 & 52.2 \pm 5.7 & 63.9 \pm 6.8 & - & 0.2238 \\ \text { Total almacenado (hojarasca + suelo) } & 82.5 \pm 8.3 & 79.6 \pm 8.3 & 78.3 \pm 8.3 & 94.8 \pm 9.5 & - & 0.1857\end{array}$

Letras diferentes indican diferencia estadística $(\mathrm{p}<0.05)$ entre tratamientos de acuerdo con la prueba LSD de Fisher. Values with the same letter are not significantly different $(\mathrm{p}>0.05)$ across treatments according fuFisher's LSD. 
La concentración de carbono en los suelos fue diferente entre los sitios $(\mathrm{F}=23.02$, $\mathrm{p}=<0.0001)$. No obstante, no se encontraron diferencias estadísticas entre tratamientos para la concentración de carbono entre $0-5 \mathrm{~cm}(\mathrm{p}=0.7787)$ y $5-20 \mathrm{~cm}$ de profundidad $(\mathrm{p}=0.7806)$. Tampoco hubo efecto de tratamiento en el contenido de carbono en el suelo (Cuadro 1). Sin embargo, se constató que la concentración de carbono a profundidad $0-5 \mathrm{~cm}(8.1 \% \pm 0.7)$ es estadísticamente superior $(\mathrm{t}=12.06, \mathrm{p}<0.0001)$ que a profundidad $5-20 \mathrm{~cm}$ $(6.2 \% \pm 0.5)$. No se encontró correlación significativa entre la concentración de carbono en las hojas y el del suelo.

Descomposición: La pérdida de peso de las hojas de I. edulis en las bolsas de descomposición entre el inicio de febrero y finales de junio de 2009 varió entre 19-23\% dependiendo del tratamiento. El modelo de regresión lineal se ajustó mejor a los datos de descomposición $\left(\mathrm{R}^{2}=0.92\right)$ en comparación con el modelo exponencial $\left(R^{2}=0.90\right)$. La curva de pérdida de peso (Fig. 4) fue muy similar entre tratamientos, las ordenadas al origen para los modelos lineales ajustados a los distintos tratamientos fueron similares $(\mathrm{P}=1.01, \mathrm{~T}=1.01, \mathrm{BS}=1.0 \mathrm{y} \mathrm{I}=0.99) \mathrm{y}$ no difirieron estadísticamente entre tratamientos $(\mathrm{p}=0.2760)$; los coeficientes $k$ de descomposición también fueron similares $(\mathrm{P}=-0.0017$, $\mathrm{T}=-0.0017, \mathrm{BS}=-0.0016$ y $\mathrm{I}=-0.0013)$ y no se encontraron diferencias entre tratamientos $(\mathrm{p}=0.4987)$. Como no hubo diferencias entre tratamiento ni para ordenadas al origen ni para pendientes, se construyó un único modelo de regresión lineal: Proporción de materia seca remanente $=1-0.0016 \times$ tiempo (días). Considerando que la descomposición es fuertemente condicionada por la precipitación, es importante mencionar que el área de estudio sufrió efectos del fenómeno metereológico El Niño y las lluvias fueron inferiores a lo esperado para ese período (IMN 2009).

\section{DISCUSIÓN}

Entender la dinámica de producción, acumulación y descomposición de hojarasca es importante para evaluar el papel de diferentes

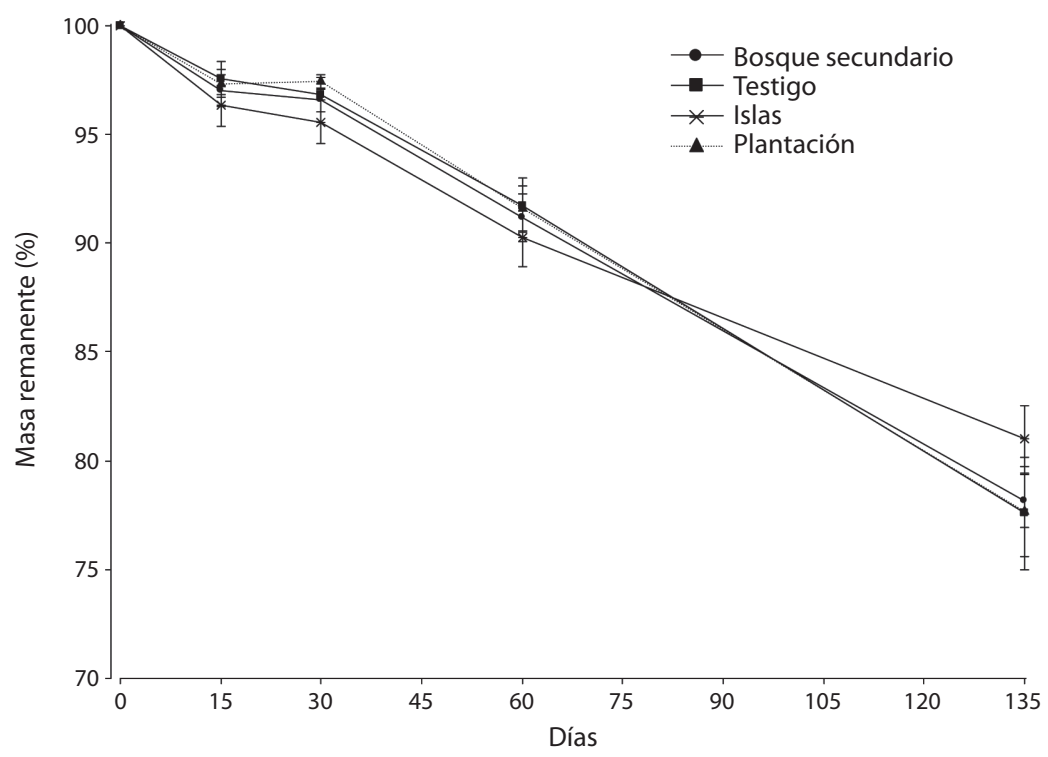

Fig. 4. Curvas de descomposición (pérdida de peso) en los tratamientos de restauración (plantación, islas, testigo y bosque secundario) en Coto Brus, Costa Rica.

Fig. 4. Decomposition curve (weight loss) in the restoration treatments (plantation, islands, control and secondary forest) in Coto Brus, Costa Rica. 
estrategias de restauración ecológica y diferentes especies en el restablecimiento de los ciclos de nutrientes y en el balance de carbono. Las plantaciones presentaron la mayor producción de hojarasca y carbono, siendo similar a los bosques secundarios jóvenes. Las plantaciones muestran el potencial para recuperar la función de producción de hojarasca rápidamente en comparación con áreas de regeneración natural y pueden proporcionar algunos de los mismos servicios ecosistémicos que los bosques secundarios más diversos (i.e. protección del suelo, almacenamiento de carbono). No obstante, aunque la función de producción pueda ser recuperada rápidamente, la calidad de la hojarasca en las parcelas, medida por la concentración de nutrientes y proporción entre carbono y nutrientes, es más alta en los bosques secundarios debido a una mayor diversidad de especies (Celentano et al. 2011); resultados similares fueron encontrados por Lugo (1992).

La calidad de la hojarasca influencia fuertemente la tasa de descomposición (Zou et al. 1995), afectando la disponibilidad de nutrientes y el proceso de sucesión (Vitousek \& Walker 1989). La hojarasca de baja calidad retrasa la descomposición y los procesos de mineralización (Wardle \& Peltzer 2007) y puede afectar a la restauración. La acumulación de hojarasca sobre el suelo es regulada por la producción y la descomposición. Las plantaciones presentan mayor acumulación de hojarasca que los otros tratamientos. Además, en este estudio, la cantidad acumulada es mayor que la producción anual en todos los tratamientos, con excepción del bosque secundario, indicando una baja tasa de descomposición. La baja tasa de descomposición es resultado del estado de degradación de los sitios, ausencia de las comunidades descomponedoras y también de la calidad del sustrato. Con el tiempo de sucesión, la tasa de descomposición tiende a aumentar y la hojarasca acumulada en el suelo disminuye (Ewel 1976). Los bosques secundarios del presente estudio presentaron menor acumulación de hojarasca sobre el suelo. Ese tratamiento tiene más edad y mayor diversidad florística.
La velocidad de descomposición es regulada por las condiciones climáticas (temperatura y precipitación; Meentemeyer 1977), por la estructura de la comunidad descomponedora (Schroth 2003) y por la calidad de la hojarasca (Xuluc-Tolosa et al. 2003). Esos factores justifican porque no se identificaron diferencias entre los tratamientos en el ensayo preliminar con bolsas de descomposición utilizando hojas de I.edulis: (1) el corto tiempo de las bolsas en el campo (135 días); (2) la reducida precipitación en el área de estudio durante el experimento debido al fenómeno climático El Niño (IMN 2009); y (3) la calidad de la hojarasca explica más variabilidad en la tasa de descomposición que las variables ambientales. Estudios adicionales por un plazo más largo y con diferentes calidades de hojarasca son necesarios para una mejor comprensión de este proceso en los sitios de restauración.

Las especies introducidas en proyectos de restauración activa juegan un papel muy importante en el restablecimiento de los procesos ecológicos. La selección de especies influencia en la tasa y ritmo de crecimiento, la producción de hojarasca y el retorno de nutrientes para el suelo (Cuevas \& Lugo 1998), y los subsecuentes procesos de restauración. Según Holl et al. (2011), la selección de especies para los tratamientos de restauración estudiados se basó en: (1) alta supervivencia en estudios previos en la región, altas tasas de crecimiento y amplio desarrollo de cubierta de copas en los primeros años y (2) disponibilidad en los viveros locales. Entre las especies introducidas, I. edulis domina el aporte de hojarasca al suelo (70\% en las plantaciones).

El Inga crece rápido y promueve la supresión de gramíneas agresivas (Holl et al. 2011), atrae pájaros (Fink et al. 2009), produce mucha hojarasca que proporciona protección del suelo, aumento de la disponibilidad de nutriente y beneficia el crecimiento de las especies maderables (Nichols et al. 2001, Nichols \& Carpenter 2006). Sin embargo, las hojas de Inga tienen una elevada fracción recalcitrante y baja tasa relativa de descomposición (Leblanc et al. 2006); lo que produce un mantillo muy 
espeso en el suelo $(3.7 \mathrm{~cm}$ en las plantaciones). Eso es bueno porque promueve una protección del suelo deseable en áreas de pendientes, formación de humus estable y potencial secuestro de carbono en el suelo a largo plazo (Leblanc et al. 2006). No obstante, esas características pueden ser indeseables para la restauración de la biodiversidad. Un mantillo de hojarasca gruesa obstruye el acceso de semillas al suelo, representa una barrera física para la emergencia de especies con semillas pequeñas, modifica las condiciones micro-ambientales promoviendo o inhibiendo la germinación y el establecimiento de plántulas (Carson \& Peterson 1990, Molofsky \& Augspurger 1992).

Esa hipótesis de trade-off entre carbono de la hojarasca y biodiversidad potencial (mayor almacenamiento de carbono de la hojarasca promueve menor biodiversidad) podrá ser comprobada o no para el corto plazo a través de la comparación entre los estudios de lluvia de semillas (Cole et al.2010) y establecimiento de plantas indígenas que se llevan a cabo en las parcelas de restauración. Será importante verificar si existe el trade-off y su temporalidad, ya que quizás a largo plazo el sistema es beneficiado con suelos más fértiles. Eso será importante para establecer recomendaciones y guiar otros proyectos de restauración en los trópicos, especialmente en ese momento donde hay un creciente interés en el carbono forestal para la mitigación del cambio climático. Áreas en restauración ecológica secuestran carbono de la atmósfera en los diferentes reservorios (biomasa aérea, biomasa subterránea, madera muerta, hojarasca y suelo). Cuantificar el secuestro de carbono en diferentes estrategias de restauración ecológica y compararlas con áreas en regeneración natural (línea base) representa una oportunidad para promover esa actividad frente a los crecientes mercados de carbono.

Las estrategias de restauración activas estudiadas (plantación mixta y en islas) aceleraron la función de producción de hojarasca y almacenamiento de carbono en comparación con las áreas en regeneración natural, las plantaciones fueron similares a los bosques secundarios más diversos. Este estudio permitió verificar que la hojarasca del suelo representa un almacenamiento de carbono que crece rápidamente durante los primeros años del proceso de restauración. La acumulación de hojarasca sobre el suelo fue superior en las plantaciones como resultado de la alta producción de hojarasca y la baja tasa de descomposición. El bosque secundario más diverso fue el unico tratamiento que presentó producción de hojarasca superior a la acumulación. Con el tiempo, se espera que la tasa de descomposición aumente en todos los tratamientos y la hojarasca acumulada en el suelo disminuya. Cinco años no fueron suficientes para detectar efectos de los tratamientos en el contenido de carbono del suelo. Los resultados de ese estudio identifican las condiciones de sitio como un factor determinante de la producción y acumulación de hojarasca y carbono, lo que evidencia el riesgo de generalizar o extrapolar resultados de estrategias de restauración.

\section{AGRADECIMIENTOS}

El proyecto de restauración en Costa Rica es financiado por la Fundación Nacional de Ciencias de los EEUU. (NSF; DEB 05-15577 para K.D. Holl y R.A. Zahawi) y la Fundación Earthwatch. Este estudio fue apoyado financieramente por ITTO (International Tropical Timber Organization), por WCS (Wildlife Conservation Society) a través del Programa de Liderazgo en Conservación (CLP) y por TNC (The Nature Conservancy) a través de la Organización para Estudios Tropicales (OET). Danielle Celentano es becaria de la Organización de los Estados Americanos (OEA). Estamos muy agradecidos por el apoyo de Juan Abel Rosales y Román Gómez Meza en el campo y de Esthefanie Daniela Rodríguez en el laboratorio. Además, agradecemos el importante soporte de Darryl Cole y Diego Delgado con logística, Gabriela Soto y Guillaume Rousseau con suelos, y Adriana Arciniegas con las correcciones del español. Finalmente, agradecemos a los finqueros en Coto Brus que permitieron el proyecto de restauración en sus tierras. También agradecemos los comentarios recibidos de tres revisores anónimos. 


\section{RESUMEN}

Estrategias de restauración tienen el potencial de acelerar el restablecimiento del ciclo de nutrientes en áreas degradadas. En este estudio, se evaluó la producción de hojarasca, su acumulación y descomposición bajo tres tratamientos: plantación (toda la superficie plantada); islas (árboles sembrados en parches de tres tamaños) y testigo (regeneración natural). También se compararon bosques secundarios jóvenes (7-9 años). Los tratamientos fueron establecidos en parcelas de 50x50m en junio 2004 en seis sitios en el sur de Costa Rica. Las especies introducidas fueron dos maderables (Terminalia amazonia y Vochysia guatemalensis) intercaladas con dos fijadoras de nitrógeno (Erythrina poeppigiana e Inga edulis). La producción total de hojarasca en la plantación y bosque secundario no difirió significativamente, fue mayor que en las islas y el testigo. La plantación presentó mayor acumulación de hojarasca sobre el suelo. Los resultados indican una gran variabilidad entre los sitios. Ambas estrategias de restauración activas aceleraron la producción y acumulación de hojarasca en comparación con la regeneración. No obstante, eso no implica la restauración del ciclo de nutrientes. La elevada acumulación de hojarasca sobre el suelo indica baja tasa de descomposición y de retorno de nutrientes al suelo.

Palabras clave: áreas degradadas, restauración, bosques tropicales, hojarasca, carbono, descomposición.

\section{REFERENCIAS}

Aronson, J., S. Milton \& J.N. Blignaut. 2007. Restoring natural capital: science, business, \& practice. Island, Washington, D.C., EEUU.

Association of Official Agricultural Chemists (AOAC). 1984. Official methods of analysis. Association of Official Agricultural Chemists, Washington, D.C., EEUU.

Bocock, K.L. \& O.J.W. Gilbert. 1957. The disappearance of leaf litter under different woodland conditions. Plant Soil 9: 197-185.

Bradshaw, A.D. 1987. Restoration: an acid test for ecology, p. 23-29. In W.R. Jordan, M.E. Gilpin \& J.E. Aber (eds.). Restoration Ecology. Cambridge, Cambridge, Inglaterra.

Carson, W.P. \& C.J. Peterson. 1990. The role of litter in an old-field community: Impact of litter quantity in different seasons on plant species richness and abundance. Oecologia 85: 8-13.

Celentano, D., R.A. Zahawi, B. Finegan, R. Ostertag, R.J. Cole \& K.D. Holl. 2011. Litterfall dynamics under different tropical forest restoration strategies. Biotropica 43: 279-287.

Céspedes, M.V., B. Finegan, B. Herrera, L.D. Delgado, S. Velásquez \& J.J. Campos. 2008. Diseño de una red ecológica de conservación entre la Reserva de Biosfera la Amistad y las areas protegidas del Área de Conservación Osa, Costa Rica. Rec. Nat. Amb. 54: 44-50.

Chazdon, R.L. 2008. Beyond deforestation: restoring forests and ecosystem services on degraded lands. Science 320: 1458-1460.

Clark, D.A., S. Brown, D.W. Kicklighter, J.Q. Cambers, J.R. Thomlinson, J. Ni \& E.A. Holland. 2001. Net primary production in tropical forests: an evaluation and synthesis of existing field data. Ecol. Appl. 11: 371-389.

Cole, R.J., K.D. Holl \& R.A. Zahawi. 2010. Seed rain under tree islands planted to restore degraded lands in a tropical agricultural landscape. Ecol. Appl. 20: $1255-1269$

Cuevas, E. \& A.E. Lugo. 1998. Dynamics of organic matter and nutrient return from litterfall in stands of ten tropical tree plantation species. Forest Ecol. Manag. 112: 263-279.

Daily, G.C., P.R. Ehrlich \& G.A Sanchez-Azofeifa. 2001. Countryside biogeography: use of human-dominated habitats by the avifauna of Southern Costa Rica. Ecol. Appl. 11: 1-13.

Di Rienzo J.A., F. Casanoves, M.G. Balzarini, L. Gonzalez, M. Tablada, C.W. Robledo. InfoStat versión 2009. Grupo InfoStat, FCA, Universidad Nacional de Córdoba, Argentina. URL http://www.infostat.com.ar

Díaz, R. \& A. Hunter. 1978. Metodología de muestreo de suelos, análisis químico de suelos y tejido vegetal e investigación en invernadero. Centro Agronómico Tropical de Investigación y Enseñanza (CATIE), Turrialba, Costa Rica.

Ewel, J.J. 1976. Litterfall and leaf decomposition in a tropical forest succession in eastern Guatemala. J. Ecol. 64: 293-308.

Food and Agriculture Organization (FAO). 2006. Global forest resources assessment 2005: progress towards sustainable forest management, FAO Forestry Paper 147. FAO, Roma, Italia. También disponible en línea: www.fao.org/forestry/fo/fra/index/jsp

Fink, R.D., C.A. Lindell, E.B. Morrison, R.A. Zahawi \& K.D. Holl. 2009. Patch size and tree species influence the number and duration of bird visits in forest 
restoration plots in southern Costa Rica. Rest. Ecol. 17: 479-488.

Holdridge, L.R., W.G. Grenke, W.H. Haheway, T. Liang \& J.J.A. Tosi. 1975. Forest environments in tropical life zones. Pergamon, Nueva York, EEUU.

Holl, K.D. \& R.B. Howarth. 2000. Paying for restoration. Rest. Ecol. 8: 260-267.

Holl, K.D. 2002. Tropical moist forest restoration, p. 539558. In M.R. Perrow \& A.J. Davy (eds.). Handbook of ecological restoration. Cambridge, Cambridge, Inglaterrra.

Holl,K.D., R.A.Zahawi, R.J.Cole, R. Ostertag \& S.Cordell. En prensa. Planting seedlings in plantations versus tree islands as a large-scale tropical forest restoration strategy. Rest. Ecol. Publicado en línea en Junio 2010 (doi: 10.1111/j.1526-100X.2010.00674.x). Versión impresa en prensa.

Instituto Meteorológico Nacional de Costa Rica (IMN). 2009. Sequía Metereológica debido a El Niño. COENOS - Boletín Enos \#25. También disponible en línea: http://www.imn.ac.cr/publicaciones/enos/BENOS252009.pdf

ITTO \& IUCN. 2005. Restoring forest landscapes: an introduction to the art and science of forest landscape restoration. Technical Series No 23. ITTO, Yokahama, Japón.

Lamb, D., P.D. Erskine \& J.A. Parrotta. 2005. Restoration of degraded tropical forest landscapes. Science 310 $1628-1632$

Lamb, D. \& D. Gilmour. 2003. Issues in forest conservation. Rehabilitation and restoration of degraded forests. International Union for Conservation of Nature and Natural Resources and World Wide Fund, Cambridge, Inglaterra.

Leblanc, H.A., P. Nygren \& R.L. McGraw. 2006. Green mulch decomposition and nitrogen release from leaves of two Inga spp. in an organic alley-cropping practice in the humid tropics. Soil Biol. Biochem. 38: 349-358.

Lugo, A.E. 1992. Comparison of tropical tree plantations with secondary forests of similar age. Ecol. Monogr. 62: $1-41$.

Mansourian, S., D. Vallauri \& N. Dudley. 2005. Forest restoration in landscapes: beyond planting trees. Springer, Nueva York, EEUU.
Millennium Ecosystem Assessment (MEA). 2005. Ecosystems and human well-being: synthesis. Island, Washington, D.C., EEUU.

Meentemeyer, V. 1977. Climatic regulation of decomposition rates of organic matter in terrestrial ecosystems, p. 779-789. In D.C. Adriano \& I.L. Brisbin (eds.). Environmental chemistry and cycling processes. U.S. Department of Energy Symposium Series. Washington, D.C., EEUU.

Meentemeyer, V., E.O. Box \& R. Thompson. 1982. World patterns and amounts of terrestrial plant litter production. BioScience 32: 125-128.

Molofsky, J. \& C. Augspurger. 1992. The effect of leaf litter on early seedling establishment in a tropical forest. Ecology 73: 68-77.

Nepstad, D., C. Uhl, C.A. Pereira \& J.M.C. da Silva. 1996. A comparative study of tree establishment in abandoned pasture and mature forest of eastern Amazonia. Oikos 76: 25-39.

Nichols, J.D., M.E. Rosemeyer, F.L. Carpenter \& J. Kettler. 2001. Intercropping legume trees with native timber trees rapidly restores cover to eroded tropical pasture without fertilization. Forest Ecol. Manag. 152: 195-209.

Nichols, J.D. \& F.L. Carpenter. 2006. Interplanting Inga edulis with Terminalia amazonia yields nitrogen benefits to the timber tree. Forest Ecol. Manag. 233: 344-351.

Oelbermann, M. \& A.M. Gordon. 2000. Quantity and quality of autumnal litterfall into a rehabilitated agricultural stream. J. Environ. Qual. 29: 603-611.

Ostertag, R., E. Marin-Spiotta, W.L. Silver \& J. Schulten. 2008. Litterfall and decomposition in relation to soil carbon pools along a secondary forest chronosequence in Puerto Rico. Ecosystems 11: 701-714.

Prach, K., R. Marrs, P. Pysek \& R. van Diggelen. 2007. Manipulation of succession, p. 121-149. In L.R. Walker, J. Walker \& R.J. Hobbs (eds.). Linking restoration and ecological succession. Springer, Nueva York, EEUU.

Pearson,T., S. Walker \& S. Brown. 2005. Sourcebook for land use, land-use change and forestry projects. World Bank \& Winrock International, Washington, D.C., EEUU.

R Development Core Team. 2010. R: A language and environment for statistical computing. R Foundation for Statistical Computing, Vienna, Austria. ISBN 
3-900051-07-0. También disponible en línea: http:// www.R-project.org

Reay, S.D. \& D.A. Norton. 1999. Assessing the success of restoration plantings in a temperate New Zealand forest. Rest. Ecol. 7: 298-308.

Rickert, E. 2005. Environmental effects of the coffee crisis: a case study of land use and avian communities in Agua Buena, Costa Rica. M.Sc. Thesis, Evergreen State College, Olympia, Washington, EEUU.

Scherer-Lorenzen, M., J.L. Bonilla \& C. Potvin. 2007. Tree species richness affects litter production and decomposition rates in a tropical biodiversity experiment. Oikos 116: 2108-2124.

Schrautzer, J., A. Rinker, K. Jensen, F. Muller, P. Schwartze \& C. DierBen. 2007. Succession and restoration of drained fens: perspectives from northwestern Europe, p.90-120. In L.R. Walker, J. Walker \& R.J. Hobbs (eds.). Linking restoration and ecological succession. Springer, Nueva York, EEUU.

Schroth, G. 2003. Decomposition and nutrient supply from biomass, p. 131-150. In G. Schroth \& F.L. Sinclair (eds.). Trees, crops and soil fertility: concepts and research methods. CAB International, Wallingford, Inglaterra.

Society for Ecological Restoration International (SERI). 2004. The primer on ecological restoration. Science and Policy Working Group, Washington, D.C., EEUU. También disponible en línea: www.ser.org

Uhl, C., R. Buschbacher \& E.A.S. Serrao. 1988. Abandoned pastures in eastern Amazonia. Patterns of plant succession. J. Ecol. 75: 663-681.

Upadhyay, V.P., U. Pandey, J.S. Singh. 1985. Effect of habitat on decomposition of standard leaf litter species. Biol. Fertil. Soils 1: 201-207.
Vitousek, P.M. \& R.L. Sanford. 1986. Nutrient cycling in moist tropical forest. Ann. Rev. Ecol. Syst. 17: 137-167.

Vitousek, P.M. \& L.R. Walker. 1989. Biological invention of Myrica faya in Hawaii: plant demography, nitrogen fixation and ecosystem effects. Ecol. Monogr. 59: $247-265$.

Walker, J. \& P. Reddel. 2007. Retrogressive succession and restoration on old landscapes, p. 1-16. In L.R. Walker, J. Walker \& R.J. Hobbs (eds.). Linking restoration and ecological succession. Springer, Nueva York, EEUU.

Wardle, D.A., K.I. Bonner \& K.S. Nicholson. 1997. Biodiversity and plant litter: experimental evidence which does not support the view that enhanced species richness affects ecosystem function. Oikos 79: 247-258.

Wardle, D.A. \& D.A. Peltzer. 2007. Abovegroundbelowground linkages, ecosystem development, and ecosystem restoration, p. 45-68. In L.R. Walker, J. Walker \& R.J. Hobbs (eds.). Linking restoration and ecological succession. Springer, Nueva York, EEUU.

Xuluc-Tolosa, F.J., H.F.M. Vester, N. Ramirez-Marcial, J. Castellanos-Albores \& D. Lawrence. 2003. Leaf litter decomposition of tree species in three successional phases of tropical dry secondary forest in Campeche, Mexico. Forest Ecol. Manag. 174: 401-412

Yarranton, G.A. \& R.G. Morrison. 1974. Spatial dynamics of a primary succession: nucleation. J. Ecol. 62: 417-428.

Zou, X., C.P. Zucca, R.B. Waide \& W.H. McDowell 1995. Long-term influence of deforestation on tree species composition and litter dynamics of a tropical rain forest in Puerto Rico. Forest Ecol. Manag. 78: $147-157$. 